\title{
New Convolutions for an Oscillatory Integral Operator on the Half-line
}

\author{
L. P. Castro ${ }^{1, a), b)}$, A. S. Silva ${ }^{1, c)}$ and N. M. Tuan ${ }^{2, d)}$ \\ ${ }^{1}$ CIDMA - Center for Research and Development in Mathematics and Applications, Department of Mathematics, \\ University of Aveiro, 3810-193 Aveiro, Portugal. \\ ${ }^{2}$ Department of Mathematics, College of Education, Viet Nam National University, G7 Build., 144 Xuan Thuy Rd., \\ Cau Giay Dist., Hanoi, Vietnam.

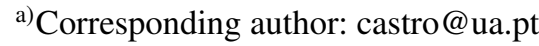

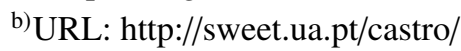 \\ c)anabela.silva@ua.pt \\ d)tuannm@hus.edu.vn
}

\begin{abstract}
The main purpose of this work is to present three new convolutions for oscillatory integral operators defined on the positive half-line and in the framework of $L^{1}$ Lebesgue spaces. Therefore, such new functions introduced by the new convolutions will have factorization properties when considering the oscillatory integral operators under consideration. Moreover, some fundamental and operational properties of the mentioned integral operator are also studied in the first part of the paper.
\end{abstract}

\section{INTRODUCTION}

Convolutions are very important operations in Functional Analysis, Integral Operators and Integral Transforms and in their applications. In fact, probably the most important property of a convolution - besides generating new functions upon previously given two or more functions - is to satisfy a factorization property which is typically intrinsically associated with one or more than one integral operators. In most of the cases, such factorization property is fundamental to solve consequent integral equations which can be characterized by those convolutions. The properties of the convolutions may also be very diverse up to the concrete definition of each convolution (cf. [1, 2, 7, 10, 15, 23, 24, 25]). The integral operators which are associated with each one of the know convolutions may have also a very distinct nature (see, e.g., [3, 4, 5, 6, 8, 9, 11, 12, 13, 14, 21, 22]). Moreover, the range of possible applications of convolutions to other sciences is also wide (cf. $[16,17,18,19,20,26])$. Here, we will introduce some new convolutions associated with oscillatory integral operators on the positive half-line. We start by defining those operators.

Let $L^{1}\left(\mathbb{R}_{+}\right)$denote the Lebesgue space of all absolutely integrable complex-valued functions on $\mathbb{R}_{+}$. For any function $f \in L^{1}\left(\mathbb{R}_{+}\right)$, we define the integral operator $S_{ \pm, \pm}$by

$$
\left(S_{ \pm, \pm} f\right)(x)=\frac{1}{\sqrt{2 \pi}} \int_{0}^{+\infty}( \pm \cos (x y) \pm \sin (x y)) f(y) d y, \quad x \in \mathbb{R}_{+}
$$

respectively.

The main aim of this work is to introduce new convolutions for the operators just presented. That is to say, in the present case, to introduce new functions, generated by two previously given functions, which will exhibit certain factorization identities when considering the above operators applied to all those functions. This will achieved in the final section of this work. 


\section{FUNDAMENTAL PROPERTIES OF THE INTEGRAL OPERATOR $S_{+,+}$}

In the present section, we will be concentrated in studying some fundamental properties of the operator $S_{+,+}$: this will be the case of some mapping properties.

\section{The Integral Operator $S_{+,+}$in the Framework of the Schwartz Space}

Let us denote by $\mathcal{S}\left(\mathbb{R}_{+}\right)$the Schwartz space on $\mathbb{R}_{+}$, and by $C_{0}^{\infty}\left(\mathbb{R}_{+}\right)$the Banach space of all continuous and infinitely differentiable functions on $\mathbb{R}_{+}$that vanish at infinity.

In this subsection, the operator $S_{+,+}$is studied in the framework of the Schwartz space. We prove that if $f \in \mathcal{S}\left(\mathbb{R}_{+}\right)$ then, $S_{+,+} f \in \mathcal{S}\left(\mathbb{R}_{+}\right)$. The following lemma shows some basic properties of the integral operator $S_{+,+}$in the Schwartz space $\mathcal{S}\left(\mathbb{R}_{+}\right)$.

\section{Lemma 1 Suppose that $f \in \mathcal{S}\left(\mathbb{R}_{+}\right)$. Then,}

(i) $S_{+,+} f$ is continuous;

(ii) $\quad\left(\frac{d^{k}}{d x^{k}} S_{+,+} f\right)(x)=\left\{\begin{array}{c}i^{k+3} S_{+,-}\left(y^{k} f\right)(x), \quad \text { if } k \text { is odd } \\ i^{k} S_{+,+}\left(y^{k} f\right)(x), \quad \text { if } k \text { is even }\end{array}, \quad k \in \mathbb{N}\right.$;

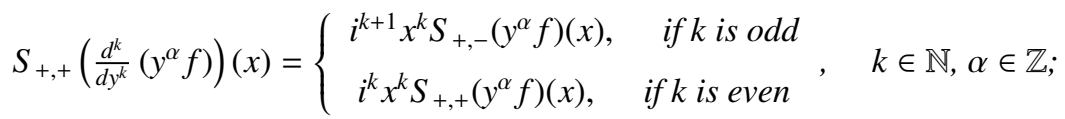

(iv) $\quad\left\|S_{+,+} f\right\|_{\infty} \leq \frac{1}{\sqrt{\pi}}\|f\|_{1}$.

Proof. (i) For any $x \in \mathbb{R}_{+}$, we have

$$
\begin{aligned}
\lim _{h \rightarrow 0}\left(S_{+,+} f\right)(x+h) & =\lim _{h \rightarrow 0} \frac{1}{\sqrt{2 \pi}} \int_{0}^{\infty}[\cos ((x+h) y)+\sin ((x+h) y)] f(y) d y \\
& =\frac{1}{\sqrt{2 \pi}} \int_{0}^{\infty} f(y) \lim _{h \rightarrow 0}[\cos ((x+h) y)+\sin ((x+h) y)] d y \\
& =\left(S_{+,+} f\right)(x) .
\end{aligned}
$$

(ii) Let us compute the first derivative of $S_{+,+} f$. We have

$$
\begin{aligned}
\left(\frac{d}{d x} S_{+,+} f\right)(x) & =\frac{1}{\sqrt{2 \pi}} \frac{d}{d x} \int_{0}^{\infty}(\cos (x y)+\sin (x y)) f(y) d y \\
& =\frac{1}{\sqrt{2 \pi}} \int_{0}^{\infty} f(y) \frac{d}{d x}(\cos (x y)+\sin (x y)) d y \\
& =\frac{1}{\sqrt{2 \pi}} \int_{0}^{\infty} y f(y)(\cos (x y)-\sin (x y)) d y \\
& =S_{+,-}(y f)(x),
\end{aligned}
$$

where the interchange of the derivative and the integral is valid since $f \in \mathcal{S}\left(\mathbb{R}_{+}\right)$. Applying the same procedure, we obtain that

$$
\begin{aligned}
\frac{d^{2}}{d x^{2}}\left(S_{+,+} f\right)(x) & =-\left[S_{+,+}\left(y^{2} f\right)\right](x), \\
\frac{d^{3}}{d x^{3}}\left(S_{+,+} f\right)(x) & =-\left[S_{+,-}\left(y^{3} f\right)\right](x), \\
\frac{d^{4}}{d x^{4}}\left(S_{+,+} f\right)(x) & =\left[S_{+,+}\left(y^{4} f\right)\right](x),
\end{aligned}
$$

and the result follows by induction. 
(iii) Integrating by parts and having into consideration that $f \in \mathcal{S}\left(\mathbb{R}_{+}\right)$, we obtain

$$
\begin{aligned}
S_{+,+}\left(\frac{d}{d y}\left(y^{\alpha} f\right)\right)(x) & =\frac{1}{\sqrt{2 \pi}} \int_{0}^{\infty} \frac{d}{d y}\left(y^{\alpha} f(y)\right)(\cos (x y)+\sin (x y)) d y \\
& =\left.\frac{1}{\sqrt{2 \pi}} y^{\alpha} f(y)(\cos (x y)+\sin (x y))\right|_{0} ^{\infty}- \\
& \frac{1}{\sqrt{2 \pi}} \int_{0}^{\infty} y^{\alpha} f(y)(-x \sin (x y)+x \cos (x y)) d y \\
& =-\frac{1}{\sqrt{2 \pi}} x \int_{0}^{\infty} y^{\alpha} f(y)(\cos (x y)-\sin (x y)) d y \\
& =-x S_{+,-}\left(y^{\alpha} f\right)(x) .
\end{aligned}
$$

With same procedure, we deduce that

$$
\begin{aligned}
& S_{+,+}\left(\frac{d^{2}}{d y^{2}}\left(y^{\alpha} f\right)\right)(x)=-x^{2} S_{+,+}\left(y^{\alpha} f\right)(x) \\
& S_{+,+}\left(\frac{d^{3}}{d y^{3}}\left(y^{\alpha} f\right)\right)(x)=x^{3} S_{+,-}\left(y^{\alpha} f\right)(x) \\
& S_{+,+}\left(\frac{d^{4}}{d y^{4}}\left(y^{\alpha} f\right)\right)(x)=-x^{4} S_{+,+}\left(y^{\alpha} f\right)(x),
\end{aligned}
$$

and the result follows by induction.

(iv) From a direct computation, it follows

$$
\begin{aligned}
\left\|S_{+,+} f\right\|_{\infty} & =\sup _{x \in \mathbb{R}_{+}}\left|\frac{1}{\sqrt{2 \pi}} \int_{0}^{+\infty}(\cos (x y)+\sin (x y)) f(y) d y\right| \\
& \leq \frac{1}{\sqrt{2 \pi}} \sup _{x \in \mathbb{R}_{+}} \int_{0}^{+\infty}|(\cos (x y)+\sin (x y))||f(y)| d y \\
& \leq \frac{1}{\sqrt{2 \pi}} \sup _{x \in \mathbb{R}_{+}} \int_{0}^{+\infty} \sqrt{2}|f(y)| d y \\
& =\frac{1}{\sqrt{\pi}}\|f\|_{1}
\end{aligned}
$$

Using the previous results, we conclude, in the following theorem, that $S_{+,+} f \in \mathcal{S}\left(\mathbb{R}_{+}\right)$for $f \in \mathcal{S}\left(\mathbb{R}_{+}\right)$.

Theorem 2 If $f \in \mathcal{S}\left(\mathbb{R}_{+}\right)$, then $S_{+,+} f \in \mathcal{S}\left(\mathbb{R}_{+}\right)$.

Proof. From Lemma $1, S_{+,+} f$ is continuous and for any pairs of integers $k$ and $\alpha$, we have that

$$
S_{+,+}\left(\frac{d^{k}}{d y^{k}}\left(y^{\alpha} f(y)\right)\right)(x)=x^{k} \frac{d^{\alpha}}{d x^{\alpha}}\left(S_{+,+} f\right)(x) .
$$

Therefore, and since $f \in \mathcal{S}\left(\mathbb{R}_{+}\right)$, we have

$$
\begin{aligned}
|x|^{k}\left|\frac{d^{\alpha}}{d x^{\alpha}}\left(S_{+,+} f\right)(x)\right| & =\left|x^{k} \frac{d^{\alpha}}{d x^{\alpha}}\left(S_{+,+} f\right)(x)\right| \\
& =\left|S_{+,+}\left(\frac{d^{k}}{d y^{k}} y^{\alpha} f\right)(x)\right| \\
& \leq \frac{1}{\sqrt{\pi}}\left\|\frac{d^{k}}{d y^{k}} y^{\alpha} f\right\|_{1}<\infty, \text { for any } x \in \mathbb{R}_{+} .
\end{aligned}
$$

Thus, $S_{+,+} f \in \mathcal{S}\left(\mathbb{R}_{+}\right)$. 


\section{The Integral Operator $S_{+,+}$in the Framework of the $L^{1}\left(\mathbb{R}_{+}\right)$Space}

We will now consider the integral operator $S_{+,+}$within the framework of the $L^{1}\left(\mathbb{R}_{+}\right)$space. With this aim, let us first recall two known results which will be useful in this study.

Theorem 3 (Lebesgue dominated convergence)

Let $\left(f_{n}\right)_{n \in \mathbb{N}}$ be a sequence of Lebesgue measurable functions defined on a Lebesgue measurable set $E$ such that the pointwise limit $f(x)=\lim _{n \rightarrow \infty} f_{n}(x)$ exists. Assume that there is an integrable $g: E \rightarrow[0, \infty]$ with $\left|f_{n}(x)\right| \leq g(x)$ for each $x \in \mathbb{R}_{+}$. Then, $f$ is integrable as is $f_{n}$ for each $n$ and

$$
\lim _{n \rightarrow \infty} \int_{E} f_{n} d \mu=\int_{E} \lim _{n \rightarrow \infty} f_{n} d \mu=\int_{E} f d \mu .
$$

Lemma 4 (Riemann Lebesgue lemma)

For a function $f$ for which the integral

$$
\int_{a}^{b}|f(x)| d x<\infty
$$

where $a$ and $b$ are real numbers, we have

$$
\begin{aligned}
& \lim _{\alpha \rightarrow \infty} \int_{a}^{b} f(x) \cos (\alpha x) d x=0 \\
& \lim _{\alpha \rightarrow \infty} \int_{a}^{b} f(x) \sin (\alpha x) d x=0
\end{aligned}
$$

or the complex form

$$
\lim _{\alpha \rightarrow \infty} \int_{a}^{b} f(x) e^{i \alpha x} d x=0 .
$$

Although, we cannot guarantee that $S_{+,+} f \in L^{1}\left(\mathbb{R}_{+}\right)$for all $f \in L^{1}\left(\mathbb{R}_{+}\right)$, in the next result, we prove that if $f \in L^{1}\left(\mathbb{R}_{+}\right)$then, $S_{+,+} f \in C_{0}^{\infty}\left(\mathbb{R}_{+}\right)$.

Theorem 5 Let $f \in L^{1}\left(\mathbb{R}_{+}\right)$. Then, $S_{+,+} f$ is a uniformly continuous function on $\mathbb{R}_{+}$and

$$
S_{+,+} f(x) \rightarrow 0 \quad \text { as } \quad x \rightarrow \infty
$$

Proof. First, we observe that

$$
\begin{aligned}
\left|S_{+,+} f(x+h)-S_{+,+} f(x)\right| & =\left|\frac{1}{\sqrt{2 \pi}} \int_{0}^{\infty}(\cos (x y+h y)+\sin (x y+h y)-\cos (x y)-\sin (x y)) f(y) d y\right| \\
& =\frac{1}{\sqrt{2 \pi}}\left|\int_{0}^{\infty} \sin \left(\frac{h y}{2}\right)\left[2 \cos \left(-\left(x+\frac{h}{2}\right) y\right)+2 \sin \left(-\left(x+\frac{h}{2}\right) y\right)\right] f(y) d y\right| \\
& \leq \frac{1}{\sqrt{2 \pi}} \int_{0}^{\infty}\left|\sin \left(\frac{h y}{2}\right)\right|\left|2 \cos \left(-\left(x+\frac{h}{2}\right) y\right)+2 \sin \left(-\left(x+\frac{h}{2}\right) y\right)\right||f(y)| d y .
\end{aligned}
$$

Since,

$$
\left|\sin \left(\frac{h y}{2}\right)\right|\left|2 \cos \left(-\left(x+\frac{h}{2}\right) y\right)+2 \sin \left(-\left(x+\frac{h}{2}\right) y\right)\right||f(y)| \leq 4\left|\sin \left(\frac{h y}{2}\right)\right||f(y)|,
$$

and

$$
\left|\sin \left(\frac{y h}{2}\right)\right||f(y)| \rightarrow 0 \quad \text { as } \quad h \rightarrow 0,
$$


from the Lebesgue's dominated convergence theorem, it follows that

$$
\int_{0}^{\infty}\left|\sin \left(\frac{y h}{2}\right)\right||f(y)| d y \rightarrow 0 \quad \text { as } \quad h \rightarrow 0 .
$$

Thus,

$$
S_{+,+} f(x+h) \rightarrow S_{+,+} f(x)
$$

and $S_{+,+} f$ is continuous on $[0, \infty]$.

The convergence of $\left(S_{+,+} f\right)(x)$ to zero, when $x \rightarrow+\infty$, follows from the Riemann Lebesgue Lemma.

\section{NEW CONVOLUTIONS}

In this section, we introduce new convolutions $\star^{(i)}(i=1,2,3)$ for the operators $S_{+,+}$and $S_{+,+}^{-}$defined in the domain $L^{1}\left(\mathbb{R}_{+}\right)$and we prove that these convolutions have a corresponding multiplicative factorization property.

In what follows, let us consider the Heaviside and the signal functions defined by

$$
H(x)= \begin{cases}1, & x>0 \\ \frac{1}{2}, & x=0 \\ 0, & x<0\end{cases}
$$

and

$$
\operatorname{sign}(x)=\left\{\begin{array}{cc}
1, & x>0 \\
0, & x=0 \\
-1, & x<0
\end{array}\right.
$$

respectively.

For $f \in L^{1}(\mathbb{R})$, let us consider the Hartley transform

$$
\left(H_{1} f\right)(x)=\frac{1}{\sqrt{2 \pi}} \int_{-\infty}^{+\infty}(\cos (x y)+\sin (x y)) f(y) d y .
$$

Moreover, let us define and use the integral operator

$$
\left(S_{+,+}^{-} f\right)(x)=\frac{1}{\sqrt{2 \pi}} \int_{0}^{+\infty}(\cos (-x y)+\sin (-x y)) f(y) d y .
$$

Definition 1 For any $f$ and $g$ in $L^{1}\left(\mathbb{R}_{+}\right)$, we define three new convolutions $\star^{(i)}$ (with $\left.i=1,2,3\right)$ by

$$
\begin{aligned}
\left(f \star^{(1)} g\right)(u) & :=\int_{0}^{\infty}\left(H(u) \ell_{0}(f(|u-t|)+f(u+t))+H(-u) \ell_{0}\left(\operatorname{sign}(u+t) \ell_{0} f(|u+t|)+f(-u+t)\right)\right) g(t) d t \\
\left(f \star^{(2)} g\right)(u) & :=\int_{0}^{\infty}\left(H(u) \ell_{0}\left(\operatorname{sign}(u-t) \ell_{0} f(|u-t|)+f(u+t)\right)+H(-u) \ell_{0}(f(|u+t|)-f(-u+t))\right) g(t) d t \\
\left(f \star^{(3)} g\right)(u) & :=\int_{0}^{\infty}\left(H(u) \ell_{0}\left(\operatorname{sign}(-u+t) \ell_{0} f(|-u+t|)+f(u+t)\right)+H(-u) \ell_{0}(f(|-u-t|)+f(-u+t))\right) g(t) d t
\end{aligned}
$$

where $u \in \mathbb{R}$ and $\ell_{0}$ are the zero extension operators from $\mathbb{R}_{ \pm}$into $\mathbb{R}$ (depending on the correspondig extension region which is clear in each application of the extension operator). 
Theorem 6 Let $f, g \in L^{1}\left(\mathbb{R}_{+}\right)$. The convolutions $\star^{(i)}(i=1,2,3)$ satisfy the following factorizations identities:

$$
\begin{aligned}
& \left(S_{+,+} f\right)(x)\left(S_{+,+} g\right)(x)=\frac{1}{4} \sqrt{\frac{2}{\pi}} r_{\mathbb{R}_{+}} H_{1}\left(f \star^{(1)} g\right)(x), \\
& \left(S_{+,+} f\right)(x)\left(S_{+,+}^{-} g\right)(x)=\frac{1}{4} \sqrt{\frac{2}{\pi}} r_{\mathbb{R}_{+}} H_{1}\left(f \star^{(2)} g\right)(x), \\
& \left(S_{+,+}^{-} f\right)(x)\left(S_{+,+}^{-} g\right)(x)=\frac{1}{4} \sqrt{\frac{2}{\pi}} r_{\mathbb{R}_{+}} H_{1}\left(f \star^{(3)} g\right)(x),
\end{aligned}
$$

where $r_{\mathbb{R}_{+}}$is the restriction operator from $\mathbb{R}$ into $\mathbb{R}_{+}$.

Proof. Let us consider the notation

$$
\operatorname{cas}(x y):=\cos (x y)+\sin (x y) .
$$

We can observe that the following identities hold true:

$$
\begin{aligned}
2 \operatorname{cas}(x y) \operatorname{cas}(x t) & =\operatorname{cas}(x(y+t))+\operatorname{cas}(x(y-t))+\operatorname{cas}(x(-y+t))-\operatorname{cas}(x(-y-t)) \\
2 \operatorname{cas}(x y) \operatorname{cas}(-x t) & =\operatorname{cas}(x(y+t))+\operatorname{cas}(x(y-t))-\operatorname{cas}(x(-y+t))+\operatorname{cas}(x(-y-t)) \\
2 \operatorname{cas}(-x y) \operatorname{cas}(-x t) & =-\operatorname{cas}(x(y+t))+\operatorname{cas}(x(y-t))+\operatorname{cas}(x(-y+t))+\operatorname{cas}(x(-y-t)) .
\end{aligned}
$$

From the last identities, we obtain the following results, just for $x \in \mathbb{R}_{+}$:

$$
\begin{aligned}
& {\left[\left(S_{+,+} f\right)\left(S_{+,+} g\right)\right](x)=} \\
= & \frac{1}{4 \pi} \int_{0}^{\infty} \int_{0}^{\infty} \operatorname{cas}(x(y+t)) f(y) g(t) d y d t+\frac{1}{4 \pi} \int_{0}^{\infty} \int_{0}^{\infty} \operatorname{cas}(x(y-t)) f(y) g(t) d y d t \\
& +\frac{1}{4 \pi} \int_{0}^{\infty} \int_{0}^{\infty} \operatorname{cas}(x(-y+t)) f(y) g(t) d y d t-\frac{1}{4 \pi} \int_{0}^{\infty} \int_{0}^{\infty} \operatorname{cas}(x(-y-t)) f(y) g(t) d y d t \\
= & \frac{1}{4 \pi} \int_{0}^{\infty} g(t) \int_{t}^{\infty} \operatorname{cas}(x u) f(u-t) d u d t+\frac{1}{4 \pi} \int_{0}^{\infty} g(t) \int_{-t}^{\infty} \operatorname{cas}(x u) f(u+t) d u d t \\
& +\frac{1}{4 \pi} \int_{0}^{\infty} g(t) \int_{-\infty}^{t} \operatorname{cas}(x u) f(-u+t) d u d t-\frac{1}{4 \pi} \int_{0}^{\infty} g(t) \int_{-\infty}^{-t} \operatorname{cas}(x u) f(-u-t) d u d t \\
= & \frac{1}{4 \pi} \int_{0}^{\infty} g(t) \int_{0}^{\infty} \operatorname{cas}(x u) H(u-t) \ell_{0} f(|u-t|) d u d t \\
& +\frac{1}{4 \pi} \int_{0}^{\infty} g(t)\left[\int_{0}^{t} \operatorname{cas}(-x u) f(-u+t) d u+\frac{1}{4 \pi} \int_{0}^{\infty} \operatorname{cas}(x u) f(u+t) d u\right] d t \\
& +\frac{1}{4 \pi} \int_{0}^{\infty} g(t)\left[\int_{0}^{t} \operatorname{cas}(x u) f(-u+t) d u+\frac{1}{4 \pi} \int_{0}^{\infty} \operatorname{cas}(-x u) f(u+t) d u\right] d t \\
& -\frac{1}{4 \pi} \int_{0}^{\infty} g(t) \int_{0}^{\infty} \operatorname{cas}(-x u) H(u-t) \ell_{0} f(|u-t|) d u d t \\
= & \frac{1}{4 \pi} \int_{0}^{\infty} g(t) \int_{0}^{\infty} \operatorname{cas}(x u) H(u-t) \ell_{0} f(|u-t|) d u d t+\frac{1}{4 \pi} \int_{0}^{\infty} g(t) \int_{0}^{\infty} \operatorname{cas}(-x u) H(-u+t) \ell_{0} f(|-u+t|) d u d t \\
& +\frac{1}{4 \pi} \int_{0}^{\infty} g(t) \int_{0}^{\infty} \operatorname{cas}(x u) f(u+t) d u d t+\frac{1}{4 \pi} \int_{0}^{\infty} g(t) \int_{0}^{\infty} \operatorname{cas}(x u) H(-u+t) \ell_{0} f(|-u+t|) d u d t \\
& +\frac{1}{4 \pi} \int_{0}^{\infty} g(t) \int_{0}^{\infty} \operatorname{cas}(-x u) f(u+t) d u d t-\frac{1}{4 \pi} \int_{0}^{\infty} g(t) \int_{0}^{\infty} \operatorname{cas}(-x u) H(u-t) \ell_{0} f(|u-t|) d u d t \\
= & \frac{1}{4 \pi} \int_{0}^{\infty} \operatorname{cas}(x u)\left[\int_{0}^{\infty}\left(H(u-t) \ell_{0} f(|u-t|)+H(-u+t) \ell_{0} f(|-u+t|)+f(u+t)\right) g(t) d t\right] d u \\
& +\frac{1}{4 \pi} \int_{0}^{\infty} \operatorname{cas}(-x u)\left[\int_{0}^{\infty}\left(H(-u+t) \ell_{0} f(|-u+t|)-H(u-t) \ell_{0} f(|u-t|)+f(u+t)\right) g(t) d t\right] d u \\
&
\end{aligned}
$$




$$
\begin{aligned}
= & \frac{1}{4 \pi} \int_{0}^{\infty} \operatorname{cas}(x u)\left[\int_{0}^{\infty}\left(\ell_{0} f(|u-t|)+f(u+t)\right) g(t) d t\right] d u \\
& -\frac{1}{4 \pi} \int_{0}^{\infty} \operatorname{cas}(-x u)\left[\int_{0}^{\infty}\left(\operatorname{sign}(-u+t) \ell_{0} f(|-u+t|)+f(u+t)\right) g(t) d t\right] d u \\
= & \frac{1}{4 \pi} \int_{0}^{\infty} \operatorname{cas}(x u)\left[\int_{0}^{\infty}\left(\ell_{0} f(|u-t|)+f(u+t)\right) g(t) d t\right] d u \\
& +\frac{1}{4 \pi} \int_{-\infty}^{0} \operatorname{cas}(x u)\left[\int_{0}^{\infty}\left(\operatorname{sign}(u+t) \ell_{0} f(|u+t|)+f(-u+t)\right) g(t) d t\right] d u \\
= & \frac{1}{4 \pi} r_{\mathbb{R}_{+}} \int_{-\infty}^{\infty} \operatorname{cas}(x u) H(u) \ell_{0}\left[\int_{0}^{\infty}(f(|u-t|)+f(u+t)) g(t) d t\right] d u \\
& +\frac{1}{4 \pi} r_{\mathbb{R}_{+}} \int_{-\infty}^{\infty} \operatorname{cas}(x u) H(-u) \ell_{0}\left[\int_{0}^{\infty}\left(\operatorname{sign}(u+t) \ell_{0} f(|u+t|)+f(-u+t)\right) g(t) d t\right] d u \\
= & \frac{1}{4 \pi} r_{\mathbb{R}_{+}} \int_{-\infty}^{\infty} \operatorname{cas}(x u)\left[\int_{0}^{\infty}\left(H(u) \ell_{0}(f(|u-t|)+f(u+t))+H(-u) \ell_{0}\left(\operatorname{sign}(u+t) \ell_{0} f(|u+t|)+f(-u+t)\right)\right) g(t) d t\right] d u .
\end{aligned}
$$

In the same way, we obtain

$$
\begin{aligned}
& {\left[\left(S_{+,+} f\right)\left(S_{+,+}^{-} g\right)\right](x)=} \\
= & \frac{1}{4 \pi} \int_{0}^{\infty} \int_{0}^{\infty} \operatorname{cas}(x(y+t)) f(y) g(t) d y d t+\frac{1}{4 \pi} \int_{0}^{\infty} \int_{0}^{\infty} \operatorname{cas}(x(y-t)) f(y) g(t) d y d t \\
& -\frac{1}{4 \pi} \int_{0}^{\infty} \int_{0}^{\infty} \operatorname{cas}(x(-y+t)) f(y) g(t) d y d t+\frac{1}{4 \pi} \int_{0}^{\infty} \int_{0}^{\infty} \operatorname{cas}(x(-y-t)) f(y) g(t) d y d t \\
= & \frac{1}{4 \pi} \int_{0}^{\infty} g(t) \int_{t}^{\infty} \operatorname{cas}(x u) f(u-t) d u d t+\frac{1}{4 \pi} \int_{0}^{\infty} g(t) \int_{-t}^{\infty} \operatorname{cas}(x u) f(u+t) d u d t \\
& -\frac{1}{4 \pi} \int_{0}^{\infty} g(t) \int_{-\infty}^{t} \operatorname{cas}(x u) f(-u+t) d u d t+\frac{1}{4 \pi} \int_{0}^{\infty} g(t) \int_{-\infty}^{-t} \operatorname{cas}(x u) f(-u-t) d u d t \\
= & \frac{1}{4 \pi} \int_{0}^{\infty} g(t) \int_{0}^{\infty} \operatorname{cas}(x u) H(u-t) \ell_{0} f(|u-t|) d u d t+\frac{1}{4 \pi} \int_{0}^{\infty} g(t) \int_{0}^{\infty} \operatorname{cas}(-x u) H(-u+t) \ell_{0} f(|-u+t|) d u d t \\
& +\frac{1}{4 \pi} \int_{0}^{\infty} g(t) \int_{0}^{\infty} \operatorname{cas}(x u) f(u+t) d u d t-\frac{1}{4 \pi} \int_{0}^{\infty} g(t) \int_{0}^{\infty} \operatorname{cas}(x u) H(-u+t) \ell_{0} f(|-u+t|) d u d t \\
& -\frac{1}{4 \pi} \int_{0}^{\infty} g(t) \int_{0}^{\infty} \operatorname{cas}(-x u) f(u+t) d u d t+\frac{1}{4 \pi} \int_{0}^{\infty} g(t) \int_{0}^{\infty} \operatorname{cas}(-x u) H(u-t) \ell_{0} f(|u-t|) d u d t \\
= & \frac{1}{4 \pi} \int_{0}^{\infty} \operatorname{cas}(x u)\left[\int_{0}^{\infty}\left(H(u-t) \ell_{0} f(|u-t|)-H(-u+t) \ell_{0} f(|-u+t|)+f(u+t)\right) g(t) d t\right] d u \\
& +\frac{1}{4 \pi} \int_{0}^{\infty} \operatorname{cas}(-x u)\left[\int_{0}^{\infty}\left(H(-u+t) \ell_{0} f(|-u+t|)+H(u-t) \ell_{0} f(|u-t|)-f(u+t)\right) g(t) d t\right] d u \\
= & \frac{1}{4 \pi} \int_{0}^{\infty} \operatorname{cas}(x u)\left[\int_{0}^{\infty}\left(\operatorname{sign}(u-t) \ell_{0} f(|u-t|)+f(u+t)\right) g(t) d t\right] d u \\
& +\frac{1}{4 \pi} \int_{0}^{\infty} \operatorname{cas}(-x u)\left[\int_{0}^{\infty}\left(\ell_{0} f(|-u+t|)-f(u+t)\right) g(t) d t\right] d u \\
= & \frac{1}{4 \pi} \int_{0}^{\infty} \operatorname{cas}(x u)\left[\int_{0}^{\infty}\left(\operatorname{sign}(u-t) \ell_{0} f(|u-t|)+f(u+t)\right) g(t) d t\right] d u \\
& +\frac{1}{4 \pi} \int_{-\infty}^{0} \operatorname{cas}(x u)\left[\int_{0}^{\infty}\left(\ell_{0} f(|u+t|)-f(-u+t)\right) g(t) d t\right] d u \\
= & \frac{1}{4 \pi} r_{\mathbb{R}_{+}} \int_{-\infty}^{\infty} \operatorname{cas}(x u) H(u) \ell_{0}\left[\int_{0}^{\infty}\left(\operatorname{sign}(u-t) \ell_{0} f(|u-t|)+f(u+t)\right) g(t) d t\right] d u \\
&
\end{aligned}
$$




$$
\begin{aligned}
& +\frac{1}{4 \pi} r_{\mathbb{R}_{+}} \int_{-\infty}^{\infty} \operatorname{cas}(x u) H(-u) \ell_{0}\left[\int_{0}^{\infty}(f(|u+t|)-f(-u+t)) g(t) d t\right] d u \\
= & \frac{1}{4 \pi} r_{\mathbb{R}_{+}} \int_{-\infty}^{\infty} \operatorname{cas}(x u)\left[\int_{0}^{\infty}\left(H(u) \ell_{0}\left(\operatorname{sign}(u-t) \ell_{0} f(|u-t|)+f(u+t)\right)+H(-u) \ell_{0}(f(|u+t|)-f(-u+t))\right) g(t) d t\right] d u .
\end{aligned}
$$

Finally,

$$
\begin{aligned}
& {\left[\left(S_{+,+}^{-} f\right)\left(S_{+,+}^{-} g\right)\right](x)=} \\
& =-\frac{1}{4 \pi} \int_{0}^{\infty} \int_{0}^{\infty} \operatorname{cas}(x(y+t)) f(y) g(t) d y d t+\frac{1}{4 \pi} \int_{0}^{\infty} \int_{0}^{\infty} \operatorname{cas}(x(y-t)) f(y) g(t) d y d t \\
& +\frac{1}{4 \pi} \int_{0}^{\infty} \int_{0}^{\infty} \operatorname{cas}(x(-y+t)) f(y) g(t) d y d t+\int_{0}^{\infty} \int_{0}^{\infty} \operatorname{cas}(x(-y-t)) f(y) g(t) d y d t \\
& =-\frac{1}{4 \pi} \int_{0}^{\infty} g(t) \int_{t}^{\infty} \operatorname{cas}(x u) f(u-t) d u d t+\frac{1}{4 \pi} \int_{0}^{\infty} g(t) \int_{-t}^{\infty} \operatorname{cas}(x u) f(u+t) d u d t \\
& +\frac{1}{4 \pi} \int_{0}^{\infty} g(t) \int_{-\infty}^{t} \operatorname{cas}(x u) f(-u+t) d u d t+\frac{1}{4 \pi} \int_{0}^{\infty} g(t) \int_{-\infty}^{-t} \operatorname{cas}(x u) f(-u-t) d u d t \\
& =-\frac{1}{4 \pi} \int_{0}^{\infty} g(t) \int_{0}^{\infty} \operatorname{cas}(x u) H(u-t) \ell_{0} f(|u-t|) d u d t+\frac{1}{4 \pi} \int_{0}^{\infty} g(t) \int_{0}^{\infty} \operatorname{cas}(-x u) H(-u+t) \ell_{0} f(|-u+t|) d u d t \\
& +\frac{1}{4 \pi} \int_{0}^{\infty} g(t) \int_{0}^{\infty} \operatorname{cas}(x u) f(u+t) d u d t+\frac{1}{4 \pi} \int_{0}^{\infty} g(t) \int_{0}^{\infty} \operatorname{cas}(x u) H(-u+t) \ell_{0} f(|-u+t|) d u d t \\
& +\frac{1}{4 \pi} \int_{0}^{\infty} g(t) \int_{0}^{\infty} \operatorname{cas}(-x u) f(u+t) d u d t+\int_{0}^{\infty} g(t) \int_{0}^{\infty} \operatorname{cas}(-x u) H(u-t) \ell_{0} f(|u-t|) d u d t \\
& =\frac{1}{4 \pi} \int_{0}^{\infty} \operatorname{cas}(x u)\left[\int_{0}^{\infty}\left(-H(u-t) \ell_{0} f(|u-t|)+H(-u+t) \ell_{0} f(|-u+t|)+f(u+t)\right) g(t) d t\right] d u \\
& +\frac{1}{4 \pi} \int_{0}^{\infty} \operatorname{cas}(-x u)\left[\int_{0}^{\infty}\left(H(-u+t) \ell_{0} f(|-u+t|)+H(u-t) \ell_{0} f(|u-t|)+f(u+t)\right) g(t) d t\right] d u \\
& =\frac{1}{4 \pi} \int_{0}^{\infty} \operatorname{cas}(x u)\left[\int_{0}^{\infty}\left(\operatorname{sign}(-u+t) \ell_{0} f(|-u+t|)+f(u+t)\right) g(t) d t\right] d u \\
& +\frac{1}{4 \pi} \int_{0}^{\infty} \operatorname{cas}(-x u)\left[\int_{0}^{\infty}\left(\ell_{0} f(|u-t|)+f(u+t)\right) g(t) d t\right] d u \\
& =\frac{1}{4 \pi} \int_{0}^{\infty} \operatorname{cas}(x u)\left[\int_{0}^{\infty}\left(\operatorname{sign}(-u+t) \ell_{0} f(|-u+t|)+f(u+t)\right) g(t) d t\right] d u \\
& +\frac{1}{4 \pi} \int_{-\infty}^{0} \operatorname{cas}(x u)\left[\int_{0}^{\infty}\left(\ell_{0} f(|-u-t|)+f(-u+t)\right) g(t) d t\right] d u \\
& =\frac{1}{4 \pi} r_{\mathbb{R}_{+}} \int_{-\infty}^{\infty} \operatorname{cas}(x u) H(u) \ell_{0}\left[\int_{0}^{\infty}\left(\operatorname{sign}(-u+t) \ell_{0} f(|-u+t|)+f(u+t)\right) g(t) d t\right] d u \\
& +\frac{1}{4 \pi} r_{\mathbb{R}_{+}} \int_{-\infty}^{\infty} \operatorname{cas}(x u) H(-u) \ell_{0}\left[\int_{0}^{\infty}(f(|-u-t|)+f(-u+t)) g(t) d t\right] d u \\
& =\frac{1}{4 \pi} r_{\mathbb{R}_{+}} \int_{-\infty}^{\infty} \operatorname{cas}(x u)\left[\int _ { 0 } ^ { \infty } \left(H(u) \ell_{0}\left(\operatorname{sign}(-u+t) \ell_{0} f(|-u+t|)+f(u+t)\right)\right.\right. \\
& \left.\left.+H(-u) \ell_{0}(f(|-u-t|)+f(-u+t))\right) g(t) d t\right] d u \text {. }
\end{aligned}
$$

The last theorem exhibits intricate factorization properties, for the new convolutions, which also relate by that identities three different integral operators: $S_{+,+}, S_{+++}^{-}$and $H_{1}$. The authors intent to continue the present study in a forthcoming paper where some of the potentialities of the three new convolutions here introduced will be applied in different contexts. 


\section{ACKNOWLEDGMENTS}

This work was supported in part by FCT-Portuguese Foundation for Science and Technology through the Center for Research and Development in Mathematics and Applications (CIDMA) of Universidade de Aveiro, within project UID/MAT/04106/2013, and by the Viet Nam National Foundation for Science and Technology Development (NAFOSTED). A. S. Silva is also supported by FCT through the postdoctoral scholarship SFRH/BPD/96763/2013.

\section{REFERENCES}

[1] P. K. Anh, L. P. Castro, P. T. Thao and N. M. Tuan, Two new convolutions for the fractional Fourier transform, Wireless Personal Communications 92(2), 623-637 (2017).

[2] P. K. Anh, L. P. Castro, P. T. Thao and N. M. Tuan, Inequalities and consequences of new convolutions for the fractional Fourier transform with Hermite weights, American Institute of Physics, AIP Proceedings 1798(1), (2017).

[3] R. N. Bracewell, The Fourier Transform and its Applications (McGraw-Hill Press, New York, 1986).

[4] G. Bogveradze and L. P. Castro, Toeplitz plus Hankel operators with infinite index, Integral Equations Operator Theory 62(1), 43-63 (2008).

[5] L. P. Castro, Regularity of convolution type operators with PC symbols in Bessel potential spaces over two finite intervals, Math. Nachr. 261-262, 23-36 (2003).

[6] L. P. Castro, R. C. Guerra and N. M. Tuan, On integral operators generated by the Fourier transform and a reflection, Memoirs on Differential Equations and Mathematical Physics 66, 7-31 (2015).

[7] L. P. Castro, L. T. Minh and N. M. Tuan, New convolutions for quadratic-phase Fourier integral operators and their applications, Mediterranean Journal of Mathematics 15: 13, 17pp. (2018).

[8] L. P. Castro and E. M. Rojas, Reduction of singular integral operators with flip and their Fredholm property, Lobachevskii Journal of Mathematics 29(3), 119-129 (2008).

[9] L. P. Castro and E. M. Rojas, Similarity transformation methods for singular integral operators with reflection on weighted Lebesgue spaces, Int. J. Mod. Math. 3(3), 295-313 (2008).

[10] L. P. Castro and S. Saitoh, New convolutions and norm inequalities, Math. Inequal. Appl 15(3), 707-716 (2012).

[11] L. P. Castro and A. S. Silva, Defect numbers of singular integral operators with Carleman shift and almost periodic coefficients, J. Math. Anal. Appl. 387, 66-76 (2012).

[12] L. P. Castro and A. S. Silva, Interplay of Wiener-Hopf and Hankel operators with almost periodic Fourier symbols on standard and variable exponent Lebesgue spaces, Annals of Functional Analysis 6(2), 49-59 (2015).

[13] L. P. Castro and F.-O. Speck, Inversion of matrix convolution type operators with symmetry, Portugalice Mathematica (N.S.) 62, 193-216 (2005).

[14] J. J. Duistermaat, Fourier Integral Operators. Reprint of the 1996 ed. (Modern Birkhuser Classics, Birkhuser, Basel, 2011).

[15] V. A. Kakichev, On the convolution for integral transforms (in Russian), Izv. ANBSSR, Ser. Fiz. Mat. 2(2), 48-57 (1967).

[16] A. Koc, H. M. Ozaktas, C. Candan and M. A. Kutay, Digital computation of linear canonical transforms, IEEE Trans. Signal Process 56(6), 2383-2394 (2008).

[17] H. M. Ozaktas, Z. Zalevsky and M. A. Kutay, The Fractional Fourier Transform with Applications in Optics and Signal Processing (Wiley, New York, 2001).

[18] S. C Pei and J. J. Ding, Relations between fractional operations and time-frequency distributions, and their applications, IEEE Trans. Signal Processing 49(8), 1638-1655 (2001).

[19] S. C. Pei and J. J. Ding, Eigenfunctions of linear canonical transform, IEEE Trans. Signal Process 50(1), 11-26 (2002).

[20] D. Przeworska-Rolewicz, "Some open questions in algebraic analysis", in Unsolved Problems on Mathematics for the 21 st Century, edited by J. M. Abe and S. Tanaka (IOS Press, 2001), pp. 109-126.

[21] S. Saitoh, Integral Transforms, Reproducing Kernels and their Applications (Pitman Research Notes in Mathematics Series 369, Longman, Harlow, 1997).

[22] S. Saitoh, Theory of reproducing kernels: applications to approximate solutions of bounded linear operator equations on Hilbert spaces, Amer. Math. Soc. Transl. 230, 107-134 (2010). 
[23] N. M. Tuan and N. T. T. Huyen, The Hermite functions related to infinite series of generalized convolutions and applications, Complex Anal. Oper. Theory 6, 219-236 (2012).

[24] N. M. Tuan and N. T. T. Huyen, Applications of generalized convolutions associated with the Fourier and Hartley transforms, Journal of Integral Equations and Applications 24, 111-130 (2012).

[25] N. M. Tuan and P. D. Tuan, Generalized convolutions relative to the Hartley transforms with applications, Sci. Math. Jpn. 70, 77-89 (2009).

[26] Q. Xiang and K. Qin, Convolution, correlation, and sampling theorems for the offset linear canonical transform, Signal, Image and Video Processing 8(3) 433-442 (2014). 